\title{
APPROXIMATION BY OPERATORS WITH FIXED NULLITY
}

\author{
RICHARD BOULDIN
}

(Communicated by John B. Conway)

\begin{abstract}
Let $T$ be a fixed operator on a complex separable Hilbert space $H$. The distance from $T$ to the operators with nullity equal to $n$, for each possible value of $n$, is determined.
\end{abstract}

1. Introduction. Let $H$ be a fixed complex separable Hilbert space. For any (bounded linear) operator $T$ on $H$ we define the nullity and deficiency, denoted nul $T$ and $\operatorname{def} T$, to be the dimensions of the kernels of $T$ and $T^{*}$, respectively. Of course, the index of $T$, denoted ind $T$, is defined to be $(\operatorname{nul} T-\operatorname{def} T)$, with $\infty-\infty$ understood to be 0 . We denote the operator norm of $T$ by $\|T\|$.

In [4] the distance from an arbitrary operator to the invertible operators (and to the Fredholm operators) was determined. This refined some results in [6] that described the closure of the invertible operators. The results and methods of [4] found application in $[\mathbf{2}, \mathbf{9}, \mathbf{1 2}]$, and other research. In this note we continue this sequence of results by determining the distance from a fixed operator $T$ to the set of operators with a given fixed nullity. The results complement [ 2 and 12]. The reader interested in operator approximations is also directed to $[\mathbf{3}, \mathbf{7}, \mathbf{8}]$, and the bibliography of [2].

Recall that the reduced minimum modulus of $T$, denoted $\gamma(T)$, is defined by $\gamma(T)=\inf \{\|T f\|:\|f\|=1, f \perp \operatorname{ker} T\}$. It is well known that the range of $T$, denoted $T H$, is closed if and only if $\gamma(T)>0$. The essential spectrum of an operator $T$, denoted $\sigma_{e}(T)$, is the set $\{z: T-z I$ is not a Fredholm operator $\}$. We define the essential minimum modulus $m_{e}(T)$ by $m_{e}(T)=\inf \left\{\lambda: \lambda \in \sigma_{e}\left(\left(T^{*} T\right)^{1 / 2}\right)\right\}$. The following enumeration of properties of $m_{e}(T)$, was developed in [4]. (i) If $E(\cdot)$ is the spectral measure for $R=\left(T^{*} T\right)^{1 / 2}$ then the smallest nonnegative $\alpha$ such that $E([\alpha, \alpha+\delta)) H$ is infinite dimensional for every positive $\delta$ is $\alpha=m_{e}(T)$. (ii) The range $T H$ is closed and nul $T$ is finite if and only if $m_{e}(T)>0$. (iii) The range $T^{*} H$ is closed and $\operatorname{def} T$ is finite if and only if $m_{e}\left(T^{*}\right)>0$. (iv) The operator $T$ is Fredholm if and only if $m_{e}(T)$ and $m_{e}\left(T^{*}\right)$ are positive. In that case, $m_{e}(T)=m_{e}\left(T^{*}\right)$.

2. Main results. To simplify subsequent notation we make the following definition.

DEFINITION. For $n$ any nonnegative integer or $\infty$ we define $\rho_{n}(T)$ by

$$
\rho_{n}(T)=\inf \{\|T-A\|: \operatorname{nul} A=n\} .
$$

Received by the editors August 1, 1986 and, in revised form, March 18, 1987.

1980 Mathematics Subject Classification (1985 Revision). Primary 47A99; Secondary 47A30, $47 \mathrm{~A} 05$.

Key words and phrases. Nullity, approximation theory, operator norm, distances in the ring of operators, essential minimum modulus, index. 
This brings us to our first theorem. The formula given here for the distance $\rho_{n}(T)$ is unlike those appearing in $[\mathbf{4}, \mathbf{2}, \mathbf{1 1}$, and 12]. It gives a significant refinement and sharpening of Proposition 4.7 in [12].

THEOREM 1. Assume that $n>\operatorname{nul} T$.

(i) We always have $m_{e}(T) \geq \rho_{n}(T) \geq \gamma(T)$. Consequently, if $m_{e}(T)=\gamma(T)$ then this common value is $\rho_{n}(T)$.

(ii) If $m_{e}(T)>\gamma(T)$ then $\rho_{n}(T)=\sup \{\lambda: \operatorname{dim} E([0, \lambda)) H<n\}$ where $U R$ is the usual polar factorization of $T$ and $E(\cdot)$ is the spectral measure for $R$.

(iii) For $n \geq 2$ and $m_{e}(T)>0, \rho_{n}(T)$ can assume any value in the interval $\left[\gamma(T), m_{e}(T)\right]$.

ProOF OF (i). For $A$ such that nul $A=n>\operatorname{nul} T$ it is immediate from the properties of $\gamma(T)$ (see [5, pp. 364-365]) that $\|T-A\| \geq \gamma(T)$. Hence, $\rho_{n}(T) \geq \gamma(T)$ and it suffices to show that $m_{e}(T) \geq \rho_{n}(T)$.

Let $U R$ be the polar factorization of $T$ with $\operatorname{ker} U=\operatorname{ker} R$. It is routine to verify that $\operatorname{ker} T=\operatorname{ker} R$ and $m_{e}(T)=m_{e}(R)$. Using property (i) of the essential minimum modulus for $R$ we construct an orthonormal sequence $\left\{f_{1}, f_{2}, \ldots\right\}$ such that $\left\{R f_{1}, R f_{2}, \ldots\right\}$ is an orthogonal set and $\left\{\left\|R f_{1}\right\|,\left\|R f_{2}\right\|, \ldots\right\}$ is a nonincreasing sequence converging to $m_{e}(R)$. One possible construction begins by letting $I_{m}=\left(m_{e}(R)+2^{-m-1}, m_{e}(R)+2^{-m}\right)$ for $m=1,2, \ldots$ and noting that $\left\{E\left(I_{1}\right), E\left(I_{2}\right), \ldots\right\}$ is pairwise orthogonal where $E(\cdot)$ is the spectral measure for $R$. To avoid contradicting property (i) we conclude that either an infinite number of the projections $\left\{E\left(I_{1}\right), E\left(I_{2}\right), \ldots\right\}$ are nonzero or else $m_{e}(R)$ is an infinite dimensional eigenvalue for $R$. In the first case, pick a unit vector $f_{k} \in E\left(I_{k}\right) H$ if $E\left(I_{k}\right) \neq 0$ and in the second, simply choose an orthonormal basis for the eigenspace corresponding to $m_{e}(R)$. Note that in the latter case it follows that $m_{e}(R)>0$ for otherwise $\operatorname{nul} T=\operatorname{nul} R=\infty$, contradicting that $n>\operatorname{nul} T$.

Let $P_{m}$ be the orthogonal projection onto $\left\{f_{m}, \ldots, f_{m+p}\right\}$ where $p+1=n-\operatorname{nul} T$ and define $A_{m}$ by $A_{m}=T\left(I-P_{m}\right)$. Note that $\operatorname{ker} A_{m}=\operatorname{ker} T \oplus P_{m} H$ and $\operatorname{nul} A_{m}=\operatorname{nul} T+(p+1)=n$. For $f$ in $H$ we have

$$
\begin{aligned}
\left\|\left(T-A_{m}\right) f\right\|^{2} & =\left\|R P_{m} f\right\|^{2}=\sum_{j=0}^{p}\left|c_{j}\right|^{2}\left\|R f_{m+j}\right\|^{2} \\
& \leq\left\|R f_{m}\right\|^{2} \sum_{j=0}^{p}\left|c_{j}\right|^{2}=\left\|R f_{m}\right\|^{2}\left\|P_{m} f\right\|^{2} .
\end{aligned}
$$

Thus,

$$
\left\|T-A_{m}\right\| \leq\left\|R f_{m}\right\| \quad \text { and } \quad \rho_{n}(T) \leq \inf _{m}\left\|T-A_{m}\right\| \leq \inf _{m}\left\|R f_{m}\right\|=m_{e}(T),
$$

which completes the proof of (i).

PROOF OF (ii). Since $m_{e}(T)$ is positive, property (ii) of the essential minimum modulus implies that the range of $T$ is closed, which implies that $\gamma(T)>0$.

Choose $A$ and $\lambda$ such that nul $A=n$ and $\operatorname{dim} E([0, \lambda)) H<n$; let $P$ denote the projection $E([0, \lambda))$. Note that

$$
\|T-A\| \geq\|(T-A)|\operatorname{ker} A\|\geq\|(I-P) R| \operatorname{ker} A\|=\|R \mid(I-P) \operatorname{ker} A\| \geq \lambda
$$


provided that $(I-P) \operatorname{ker} A$ is nontrivial. Clearly nul $(I-P)<n=\operatorname{nul} A$ and thus $(I-P) \operatorname{ker} A$ must be nontrivial. From the inequality deduced above it follows that $\rho_{n}(T) \geq \lambda$ and

$$
\rho_{n}(T) \geq \mu \equiv \sup \{\lambda: \operatorname{dim} E([0, \lambda)) H<n\} .
$$

Now we prove the inequality opposite to $(*)$. From the usual properties of the spectral measure it follows that $\operatorname{dim} E([0, \mu+1 / k)) H \geq n$. Choose $M_{k}$ to be a subspace of $E([0, \mu+1 / k)) H$ that contains $E([0, \mu)) H$ and such that $\operatorname{dim} M_{k}=$ $n$. Let $P_{k}$ be the orthogonal projection onto $M_{k}$ and note that nul $T\left(I-P_{k}\right)=$ $\operatorname{dim} M_{k}=n$. If $A_{k}$ is $T\left(I-P_{k}\right)$ then $\left\|T-A_{k}\right\| \leq \mu+1 / k$ and $\rho_{n}(T) \leq \inf \left\|T-A_{k}\right\| \leq$ $\mu$, as desired.

ProOF OF (iii). If $\gamma(T)=m_{e}(T)$ then there is nothing to prove. Choose two positive numbers $a$ and $c$ such that $a<c$; choose $b$ such that $a \leq b \leq c$. Choose $n$ to be some integer such that $n \geq 2$. We shall construct $T$ such that $\gamma(T)=a$, $\rho_{n}(T)=b$, and $m_{e}(T)=c$. Of course, $m_{e}(T)>0$ implies that the range of $T$ is closed, which implies that $\gamma(T)>0$. Define $T$ to be the diagonal operator with the first $n-1$ entries equal to $a$, the next two entries equal to $b$ and all of the remaining entries equal to $c$. Note that $T=R$. Let $D$ be the diagonal operator with the first $n$ entries equal to 0 , the $n+1$ entry equal to $b$, and all other entries equal to $c$. Clearly we have $\|T-D\|=b$ and $\operatorname{ker} D=n$; thus, $\rho_{n}(T) \leq b$.

Let $P$ be the orthogonal projection onto $\operatorname{ker}(T-a)$ and let $A$ be any operator with nul $A=n$. Note that

$$
\|T-A\| \geq\|(I-P) R|\operatorname{ker} A\|=\| R|(I-P) \operatorname{ker} A\| \geq b ;
$$

the last inequality follows because $(I-P) \operatorname{ker} A$ must be nontrivial. It follows that $\rho_{n}(T) \geq b$; this inequality and the preceding paragraph show that $\rho_{n}(T)=b$, as desired. It is routine to verify that $\gamma(T)=a$ and $m_{e}(T)=c$.

In the next theorem the formula for $\rho_{n}(T)$ resembles previously obtained distance formulas. This theorem provides a notable sharpening and simplification of Proposition 3.10 in [12]. It gives an interesting contrast to Theorem 12.2 of [2, p. 146].

THEOREM 2. Assume that $n<\operatorname{nul} T$.

(i) If $n \geq \operatorname{ind} T$ then $\rho_{n}(T)=0$.

(ii) If $n<$ ind $T$ then $\rho_{n}(T)=m_{e}\left(T^{*}\right)$.

ProOF OF (i). Note that def $T \geq \operatorname{nul} T-n$. Let $\left\{e_{1}, e_{2}, \ldots\right\}$ be an orthonormal basis for $\operatorname{ker} T$ and note that this basis could be finite or infinite. Let $\left\{f_{1}, f_{2}, \ldots\right\}$ be an orthonormal basis for $\operatorname{ker} T^{*}$ and note that it is infinite if the basis for $\operatorname{ker} T$ is infinite. Define $A_{k}$ by

$$
\begin{aligned}
A_{k} e_{j} & =0, \quad j=1,2, \ldots, n, \\
A_{k} e_{n+j} & =(1 / k) f_{j}, \quad j=1,2, \ldots, \\
A_{k} \mid(\operatorname{ker} T)^{\perp} & =T \mid(\operatorname{ker} T)^{\perp} .
\end{aligned}
$$

It is routine to verify that nul $A_{k}=n$ and $\left\|T-A_{k}\right\|=1 / k$. This proves (i).

PROOF OF (ii). This part of the proof requires the consideration of cases. Using the methods of [4] to refine Theorem 12.2 in [2, p. 146], Wu shows in [12, Theorem 3.1] that $\rho_{n}(T) \leq \max \left\{m_{e}(T), m_{e}\left(T^{*}\right)\right\}$. A folklore result, which is proved in [11], 
indicates that ind $A=\operatorname{ind} T$ whenever $\|T-A\|<m_{e}(T)$. This result implies that $\rho_{n}(T) \geq \max \left\{m_{e}(T), m_{e}\left(T^{*}\right)\right\}$. Thus, we have $\rho_{n}(T)=\max \left\{m_{e}(T), m_{e}\left(T^{*}\right)\right\}$ and we need only argue that the right side of the preceding equation equals $m_{e}\left(T^{*}\right)$. Property (iv) of the essential minimum modulus gives the desired conclusion whenever $m_{e}\left(T^{*}\right)$ is positive. Because ind $T$ exceeds $n$, which is a nonnegative integer, we conclude that nul $T>\operatorname{def} T$. Thus, $\operatorname{def} T$ is finite and $m_{e}\left(T^{*}\right)=0$ implies that the range of $T^{*}$ is not closed according to property (iii) of the essential minimum modulus. Thus, the range of $T$ is not closed and $m_{e}(T)=0$ according to property (ii). This proves that

$$
\max \left\{m_{e}(T), m_{e}\left(T^{*}\right)\right\}=0=m_{e}\left(T^{*}\right)
$$

and concludes the proof of (ii).

By taking adjoints and using various elementary results we can restate Theorems 1 and 2, respectively, as formulas for approximation by operators with fixed deficiency.

COROLlaRY. Let $\eta_{n}(T)=\inf \{\|T-A\|: \operatorname{def} A=n\}$ and assume that $n>\operatorname{def} T$.

(i) $m_{e}\left(T^{*}\right) \geq \eta_{n}(T) \geq \gamma(T)$.

(ii) If $m_{e}\left(T^{*}\right)>\gamma(T)$ then $\eta_{n}(T)=\sup \{\lambda$ : $\operatorname{dim} E([0, \lambda)) H<n\}$ where $E(\cdot)$ is the spectral measure for $\left(T T^{*}\right)^{1 / 2}$.

(iii) For $n \geq 2$ and $m_{e}\left(T^{*}\right)>0, \eta_{n}(T)$ can assume any value in the interval $\left[\gamma(T), m_{e}\left(T^{*}\right)\right]$.

COROLlaRY. Assume that $n<\operatorname{def} T$.

(i) If $n \geq-\operatorname{ind} T$ then $\eta_{n}(T)=0$.

(ii) If $n<-$ ind $T$ then $\eta_{n}(T)=m_{e}(T)$.

\section{REFERENCES}

1. T. Ando, T. Sekiguchi, and T. Suzuki, Approximation by positive operators, Math. Z. 131 (1973), 273-282.

2. C. Apostol, L. A. Failkow, D. A. Herrero and D. Voiculescu, Approximation of Hilbert space operators, vol. II, Pitman, Boston, Mass., 1984.

3. R. Bouldin, Positive approximants, Trans. Amer. Math. Soc. 177 (1973), 391-403.

4. __ The essential minimum modulus, Indiana Univ. Math. J. 30 (1981), 513-517.

5. J. B. Conway, A course in functional analysis, Springer-Verlag, New York, 1985.

6. J. Feldman and R. V. Kadison, The closure of the regular operators in a ring of operators, Proc. Amer. Math. Soc. 5 (1954), 909-916.

7. P. R. Halmos, Ten problems in Hilbert space, Bull. Amer. Math. Soc. 76 (1970), 887-933.

8. __ Positive approximants of operators, Indiana Univ. Math. J. 21 (1972), 951-960.

9. S. Izumino and Y. Kato, The closure of invertible operators on a Hilbert space, Acta Sci. Math. (Szeged) 49 (1985), 321-327.

10. D. D. Rogers, Normal spectral approximation, Ph.D. thesis, Indiana Univ., 1975.

11. __ Approximation by unitary and essentially unitary operators, Acta Sci. Math. (Szeged) 39 (1977), 141-151.

12. P. Y. Wu, Approximation by invertible and noninvertible operators, J. Approximation Theory (to appear).

Department of Mathematics, University of Georgia, Athens, Georgia 30602 